\title{
Evaluation of Green Synthesis of Ag Nanoparticles Using Eruca sativa and Spinacia oleracea Leaf Extracts and Their Antimicrobial Activity
}

\author{
Ibrahim A. Alaraidh ${ }^{1}$; Mohamed M. Ibrahim ${ }^{1,2,}$; Gehan A. El-Gaaly ${ }^{3}$ \\ ${ }_{1}^{1}$ Department of Botany and Microbiology, Science College, King Saud University, Riyadh, Saudi Arabia \\ 2 Department of Botany and Microbiology, Faculty of Science, Alexandria University, Alexandria, Egypt \\ 3 Department of Botany and Microbiology, Science College (Female Center for Scientific and Medical College), King Saud University, Riyadh, Saudi Arabia \\ ${ }^{*}$ Corresponding author: Mohamed M. Ibrahim, Botany and Microbiology Department, Faculty of Science, Alexandria University, Alexandria, Egypt. Tel: +96-614829053, Fax: +96- \\ 65512088397, E-mail: m_ibramim2004@yahoo.com
}

Received: May 24, 2013; Revised: October 5, 2013; Accepted: October 22, 2013

\begin{abstract}
Background: Nano-biotechnology is considered as one of the main vigorous fields of research in new material science. Recently, biosynthetic methods employing both biological microorganisms such as bacteria and fungus or plant extracts have developed rapidly as a trouble-free and feasible choice to obtain nanomaterials alternative to more complex chemical synthetic procedures. The particular distinctiveness like size, allocation and shape give the nanoparticles different properties from bulk material.

Objectives: Eruca sativa and Spinacia oleracea plants were evaluated for their extra cellular potential synthesis of silver nanoparticles and their bactericidal impact on Streptococcus pneumoniae and Pseudomonas aeruginosa.

Materials and Methods: Aqueous solutions of $\mathrm{AgNO}_{3}$ were mixed with plant extracts. Transmission electron microscopy (TEM) was used to characterize the morphology of the nanoparticles obtained from plant extracts. Energy dispersiveX-ray(EDX) spectrometer established the existence of elemental sign of the silver and homogenous allocation of silver nanoparticles. Diffraction by using X ray (XRD) analysis for the formed AgNPs revealed spherical plus cubical structures with different planes ranging between 111 to 311 planes. Scanning electron microscopy (SEM) was used to characterize the morphology of the nanoparticles obtained from plant extracts.

Results: The antibacterial action of AgNPs against human pathogens, Streptococcus pneumoniae and Pseudomonas aeruginosa was recognized. Our work showed a rapid, eco-safe and suitable method for the synthesis of AgNPs from Eruca sativa and Spinacia oleracea leaf extracts and can be used for pharmaceutical and other biomedical applications.

Conclusions: It can be suggested that AgNPs show effective antibacterial properties owing to their exceptionally big exterior region, which provides superior contact with microorganisms and its interactions with bacteria are and localized on the membrane of the organism.
\end{abstract}

Keywords: EDX; Eruca; Green Biotechnology; Nanoparticles; Spinacia; TEM

\section{Background}

Nano-biotechnology is considered as one of the main vigorous fields of research in new material science. Recently, biosynthetic methods employing both biological microorganisms such as bacteria (1) and fungus (2) or plants extract $(2,3)$, have developed rapidly as a troublefree and feasible choice to obtain nanomaterials alternative to more complex chemical synthetic procedures. Their particular distinctiveness like size, allocation and shape give the nanoparticles different properties from the bulk material (4).

Current nanotechnology developments have led to nanomedicine, a new field which includes many diagnostic and therapeutic applications involving nanomaterials and nanodevices (5). Synthesis of nanoparticles using plant extracts supplies progression more than chemical and physical methods as it is cost effective, environmentally safe, simply scaled up for great range production and in this process there is no requirement to use high pressure, power, temperature and poisonous chemicals (6).

Green production of AgNPs requires three main processes, which have to be checked based on green chemistry phenology, including (1) the assortment of solvent medium, (2) the choice of environmentally kind reducing agent, and (3) the choice of safe substances for the steadiness of AgNPs.

Bio reduction of silver and gold ions to yield metal nanoparticles using plant extract $(3,7)$, Geranium leaf broth (2), Neem leaf broth (8) lemongrass extract (9), Tamarind leaf extract (10) and Aloe vera plant extracts (3) have been reported. Shankar et al. (8) reported on the synthesis of clean spiky nanoparticles of silver and gold by

Implication for health policy/practice/research/medical education: This study represents the application of Nano technology in biological sciences.

Copyright @ C 2014, National Institute of Genetic Engineering and Biotechnology; Published by Kowsar Corp. This is an open-access article distributed under the terms of the Creative Commons Attribution License, which permits unrestricted use, distribution, and reproduction in any medium, provided the original work is properly cited. 
the reduction of $\mathrm{Ag}^{+}$and $\mathrm{Au}^{3+}$ ions by Neem (Azadirachta indica) leaf broth. Most of the reported green synthesis methods using plants took more than 1 hour for the formation of colloidal silver $(11,12)$.

Nanobiotechnology has enhanced the production of minor AgNPs with little toxic effect to human and more effectiveness alongside bacteria (13-16). Furthermore, nanoparticles are alternatives to antibiotics allowing better action against multidrug opposing bacteria and consequently plant-derived nanoparticles have been proved better than other methods (17-19). The method of the AgNPs antibacterial action has been efficiently explained in conditions of their interaction with cell membranes of bacteria by troubling its permeability and respiratory role $(20,21)$.

\section{Objectives}

In this study, we used aqueous plant extracts of Eruca sativa and Spinacia oleracea leaves for the creation of silver nanoparticles and studied their antiseptic activity against Streptococcus pneumoniae and Pseudomonas aeruginosa.

\section{Materials and Methods}

\subsection{Plant Material and Synthesis of the Extract}

Fresh leaves of Eruca sativa and Spinacia oleracea were used to make the extract. Twenty-five grams of fresh green leaves were systematically washed with distilled water followed by double distilled water to remove dust particles and other pollutants. Next, the plant substance was chopped into fine slices and placed in a clean $250 \mathrm{~mL}$ Erlenmeyer conical flask and $100 \mathrm{~mL}$ of germ-free double distilled water was added and the flask was incubated on a sand bath at $60^{\circ} \mathrm{C}$ for 30 minutes to facilitate the formation of aqueous leaf extract. The extract was then filtered using Whitman No. 1 filter paper. The plant extract was used for the synthesis of AgNPs and the extract was stored at $4{ }^{\circ} \mathrm{C}$ for further use.

\subsection{Preparation of $1 \mathrm{mM}$ Silver Nitrate Solution}

For the preparation of $1 \mathrm{mM}$ Silver nitrate $\left(\mathrm{AgNO}_{3}\right), 0.02$ $\mathrm{g}$ of $\mathrm{AgNO}_{3}$ was added to $100 \mathrm{~mL}$ of double distilled water. The solution was mixed thoroughly and stored in an amber colored bottle in order to prevent auto oxidation of silver.

\subsection{Synthesis of Silver Nano Particles}

For the production of $5 \%$ plant mediated AgNPs; $5 \mathrm{~mL}$ of plant extract was added to $95 \mathrm{~mL}$ of $1 \mathrm{mM}$ silver nitrate solution and incubated on a sand bath at $60^{\circ} \mathrm{C}$ for 30 minutes, after which a color change was observed. This indicates preliminary confirmation for the formation of AgNPs. The brown color formation indicates that the Ag-
NPs were synthesized from the herbs extract and at this point the solutions were centrifuged at $5000 \mathrm{rpm}$ (Hettich EBA20S Portable Centrifuge) for 10 minutes in order to obtain the pellet which is used for further study.

\subsection{Transmission Electron Microscope (TEM)}

TEM measurements and photographs were carried out on a JEOL-TEM 1200 EX II Transmission Electron Microscope at the Faculty of science in Alexandria University (Alexandria, Egypt). The sample was dried in order to take the photograph.

\subsection{Energy-Dispersive X-Ray Spectrometer (EDX) Analysis}

AgNPs were cut-off by centrifuging $20 \mathrm{~mL}$ of solution in water including AgNPs for 10 minutes at 15,000 rpm (Hettich EBA20S Portable Centrifuge). The pellets were obtained and dehydrated in an oven at $50^{\circ} \mathrm{C}$ to remove water. The AgNPs was obtained in powder form and was used for EDX investigation. To perform EDX investigation, the leaf extract AgNPs were dehydrated and full covered on to carbon layer. EDX investigation was then performed using electron microscope (SEM) set with EDX.

EDX can be used to confirm the composition and distribution of the nanoparticles through spectrum and elemental mapping by using an EDX spectrometer incorporated into a scanning electron microscopy (SEM) system.

\subsection{UV-Visual Observation}

UV-Vis absorption spectra were measured using LKB spectrophotometer.

\subsection{Microorganisms}

The evaluation of antibacterial action was done using various strains. The following microorganisms were used:Streptococcus pneumoniae (Thermo Fisher Scientific, AS Polyvalent 2 R671260, Waltham; USA) and Pseudomonas aeruginosa (Thermo Fisher Scientific, Set R670372, Waltham; USA). The microbial cultures were maintained by the Department of Botany and Microbiology, College of Science, King Saud University, Riyadh, Saudi Arabia.

\subsection{Antibacterial Activity Study}

Antiseptic action of the synthesized AgNPs was performed using the agar well diffusion examination process (22). For this technique, disinfected Mueller-Hinton Agar plates were prepared. Pathogenic bacteria used in the current research were spread on the agar plates by sterile cotton wipes. The plates were allowed to dry and a sterile well-cutter with $5.0 \mathrm{~mm}$ diameter was used to bore wells in the agar plates. Subsequently, $50 \mu \mathrm{L}$ of the synthesized nanoparticle suspension (mass concentration = $0.02 \mu \mathrm{g} . \mu \mathrm{L}^{-1}$ ) was introduced into wells of the inoculated Mueller-Hinton Agar plates. Another two concentrations, 
10 and $25 \mu \mathrm{L}$ of the synthesized Ag-nanoparticles were used and introduced into wells with Agar. The plates containing the bacterial and AgNPs were left for 1 hour to allow diffusion to take place and then incubated at $37^{\circ} \mathrm{C}$ for 24 hours, and then observed for indication of zones of inhibition, which were clear regions around the wells (23). The length of such inhibition zones was calculated using a metre ruler, and the significant value for each type of bacteria was documented in millimeters.

\section{Results}

When plant leaf extract was uniformed in aqueous solution of $1 \mathrm{mM}$ AgNO3, the reduction of Ag was measured by UV-Vis spectrum of the process. The reduction of $\mathrm{AgNO}_{3}$ into AgNPs during contact with plant extracts was followed by a slow raise in color progress from clear yellow to brown (Figure 1). The absorption spectra of Ag-
NPs formed, showed the creation of AgNPs with almost $100 \%$ plant reduction of $\mathrm{Ag}$ ions as supported by qualitative testing of supernatant after the decontamination of silver nanoparticles by heat (Figure 1).

SEM examination was performed to recognize the topology, surface morphology and the dimension of the AgNPs, which revealed production of higher density poly dispersed round AgNPs of different sizes that varied between 4.97-14.4 $\mathrm{nm}$ for Eruca sativa and from 2.31-16.07 $\mathrm{nm}$ for Spinacia oleracea. The majority of the AgNPs were collected and only some of them were spread and examined under SEM (Figure $2 \mathrm{~A}$ and B). From the images, the nanoparticles appear to be aggregated and the surface of the aggregates is rough. Scanning electron micrograph (SEM) was equipped with energy dispersive spectroscopy(EDX). The presence of silver was confirmed from the Ag peak obtained from the EDX spectrum as shown in Figure $2 \mathrm{C}$ and D.
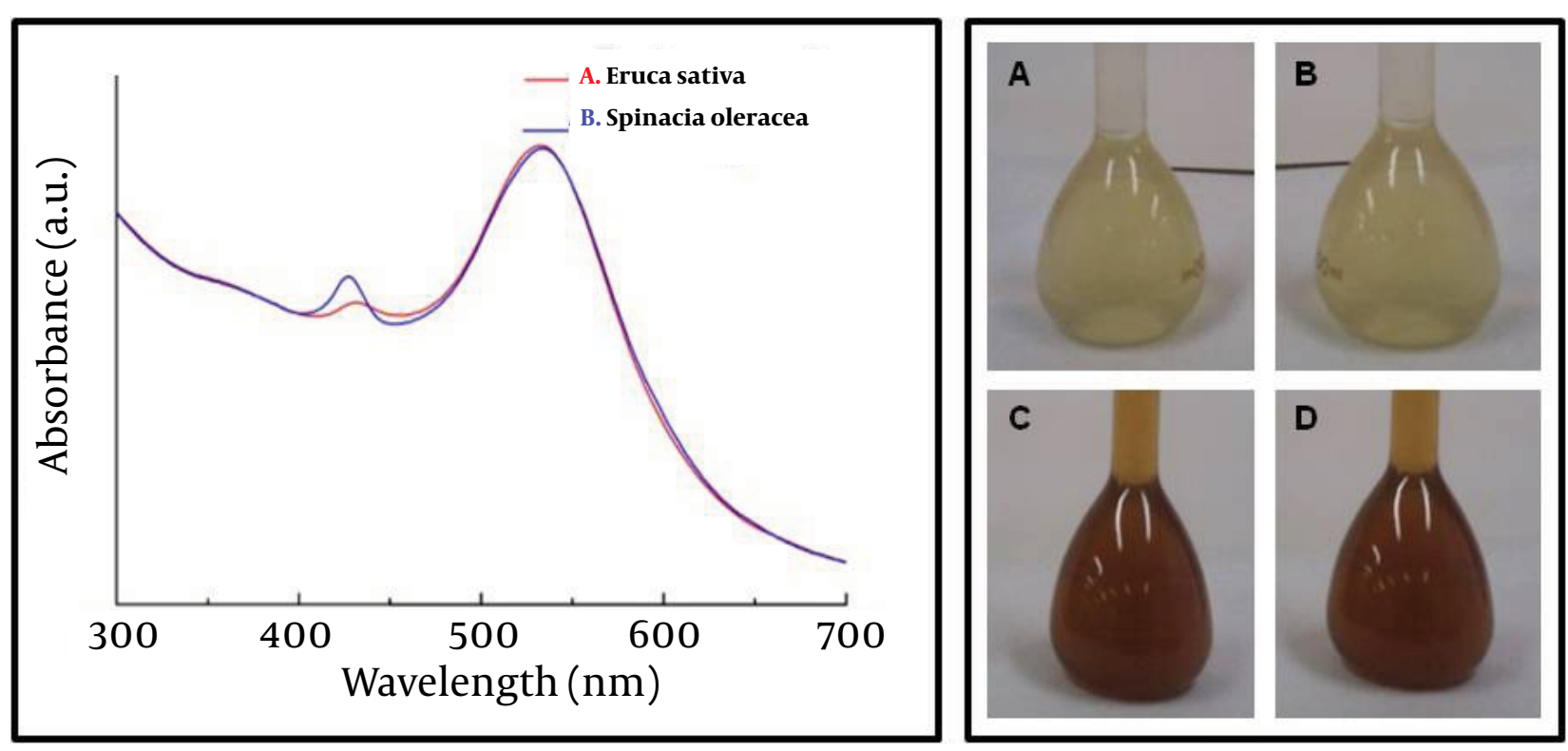

Figure 1. UVa Vis spectra showing absorption recorded as a function of 1mM AgNO3 with aqueous leaf extract of Eruca sativa and Spinacia oleracea. Color development with time for Eruca sativa (A and C) and (B and D) Spinacia oleracea.

Examination of AgNPs by energy dispersive X-ray (EDX) spectrometer established the existence of elemental indication of the $\mathrm{Ag}$ and homogenous distribution of AgNPs (Figure $2 \mathrm{C}$ and $\mathrm{D}$ ). The pointed sign peak of Ag powerfully established the reduction of $\mathrm{AgNO}_{3}$ to AgNPs. The upright axis expresses the number of X-ray counts while the parallel axis shows energy in KeV. Detection lines for the main release energy for Ag were clarified and these communicate with peaks in the spectrum, thus giving affirmation that $\mathrm{Ag}$ has been properly recognized and present in the solution. The appearance of some other minute signals may be due to the thin film made on the glass slide taken for the EDX.

The X-ray diffraction patterns (XRD) of the AgNPs formed by the leaf extract of both Eruca sativa and Spinacia oleracea were additionally established by the distinguished peaks examined in the XRD image (Figure 3). The XRD pattern showed four intense peaks $\left(27.94^{\circ}, 32.36\right.$ , $46.36^{\circ}$ and $56.28^{\circ}$ ) in the full spectrum of $2 \theta$ value between $20^{\circ}$ and $70^{\circ}$.

Transmission electron micrographs give the closed view of spherical Ag nanoparticle and indicate that they are also spherical (Figure 4). AgNPs covered with minor particles were not well estranged from each other in the nanotriangles in the extent ranges, $45-60 \mathrm{~nm}$. This is due to the occurrence of small crystal and hexagonal particles of about 10-25 nm (width) on the triangular face. 


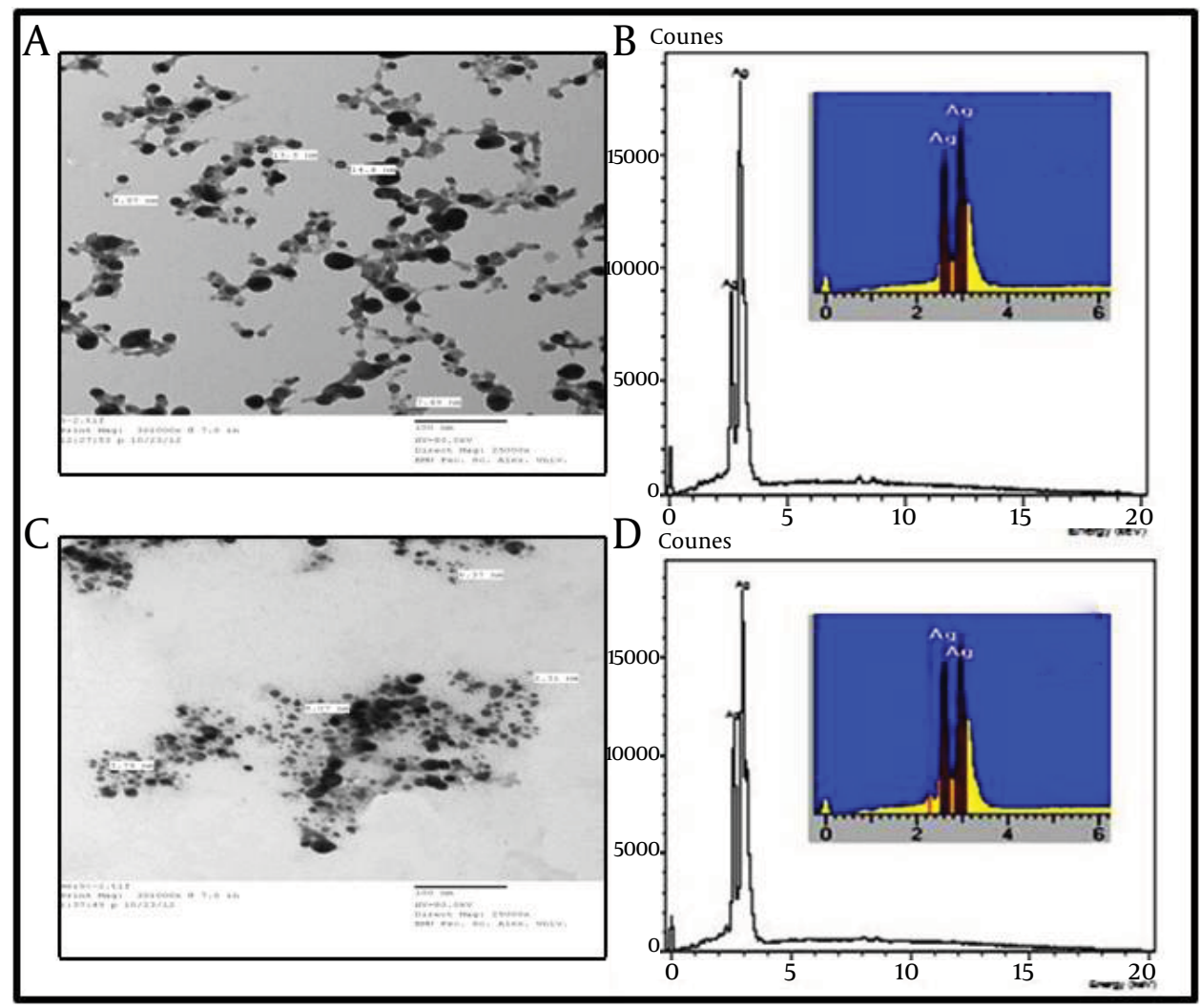

Figure 2. SEM images of AgNPs from leaf extract of (A) Eruca sativa and (B) Spinacia oleracea. Analysis of energy dispersive X-ray (EDX) spectrometer of the particles formed by leave extracts of (C) Eruca sativa and (D) Spinacia oleracea.

The antibacterial activity for silver nanoparticles was determined with gram-positive bacterial strains, such as Streptococcus pneumonia and gram-negative bacterial strains such as Pseudomonas aeruginosa. The inhibition zone caused by $50 \mu \mathrm{L}$ of synthesized silver nanoparticles in Eruca sativa leaves extract reached about $2.5 \mathrm{~cm}$ for gram-positive strains (Streptococcus pneumonia) and 2.8 $\mathrm{cm}$ for Eruca sativa and $3.2 \mathrm{~cm}$ for Spinacia oleracea. On the other hand, lower effect by Ag NPS on negative strains Pseudomonas aeruginosa was observed (Figure 5). Lower antibacterial activity was observed for both 10 and $25 \mu \mathrm{L}$ of gram-positive and gram-negative bacteria for both plants (Data not shown).

\section{Discussion}

It is well-known that silver nanoparticles exhibit yellowish brown color in aqueous solution (19). The organized samples show considerable alterations in the color of Eruca sativa and Spinacia oleracea leaves extracts. This examination was further established by UV-Vis spectrophotometer as shown in Figure 1. According to our results, it could be confirmed that Eruca sativa and Spinacia oleracea leaves extracts display a reducing potential in terms of production rate and change to silver nanoparticles.
A small absorption band at $435 \mathrm{~nm}$ shows the absorption spectra of the produced sample ( $0.1 \mathrm{~mL}$ sample). This band grew and blue changed from 435 to $495 \mathrm{~nm}$ with increasing time. This band is related to absorption by AgNPs in the visible area (380-450 $\mathrm{nm}$ ) due to the occurrence of exterior plasmon sensations (24). The raise of the peaks strength show that the absorption of AgNPs was promoted (16). The symmetric and thin absorption peak indicates the fine size allocation of the AgNPs. The peak change of greatest absorption wavelength clarifies that the dimension of AgNPs decreases with increasing time. Here, we suggest that with prolonged time, the rate of nuclei impulsive rises and a high number of nuclei are created throughout the nucleation rupture. Thus, the amount of ending particles rises, and the variance of particle dimension therefore lowers. This examination obviously indicates the occurrence of AgNPs reduction using Eruca sativa and Spinacia oleracea leaves extracts.

The characterization of AgNPs by EDX outline evidenced physically powerful signals for Ag atoms as shown in Figure 2. The EDX pattern clearly shows that the Ag nanoparticles are crystalline in nature, which is caused by the reduction of silver ions using Eruca sativa and Spinacia oleracea leaf extracts. 

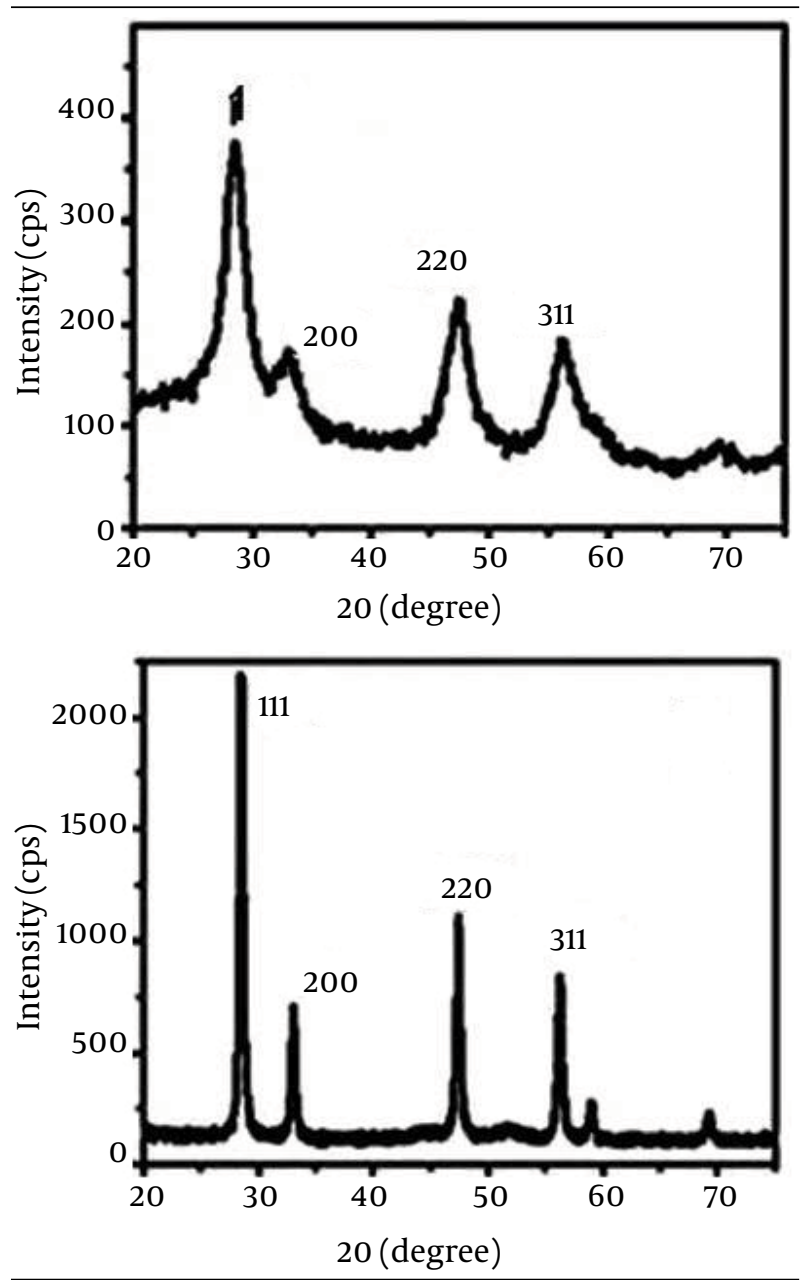

Figure 3. XRD Analysis

The X-ray diffraction (XRD) has been confirmed to be an important study means to show the creation of AgNPs and the crystal formation of the prepared AgNPs and to estimate the crystalline particle dimension.

The antibacterial activity of biologically synthesized silver nanoparticles from leaf extracts of both Eruca sativa and Spinacia oleracea plants was evaluated against $S$. pneumonia and S. aeruginosa, showing more effective bactericidal activity in opposition to gram-negative bacteria than gram-positive. It could be suggested that AgNPs show effective antibacterial properties owing to their exceptionally big exterior region, which provides superior contact with microorganisms and its interactions with bacteria are and localized on the membrane of the organism. Our results are consistent with Shrivastava et al. (25) who reported that the silver nanoparticles have an antimicrobial effect on S. aureus and E. coli. Similarly, Kim et al. (26) proved bactericidal activities of AgNPs in opposition to E. coli and S. aureus. They suggested that the cause was quantity requirements and was more prominent in opposition to gram-negative organisms than gram-positive ones.
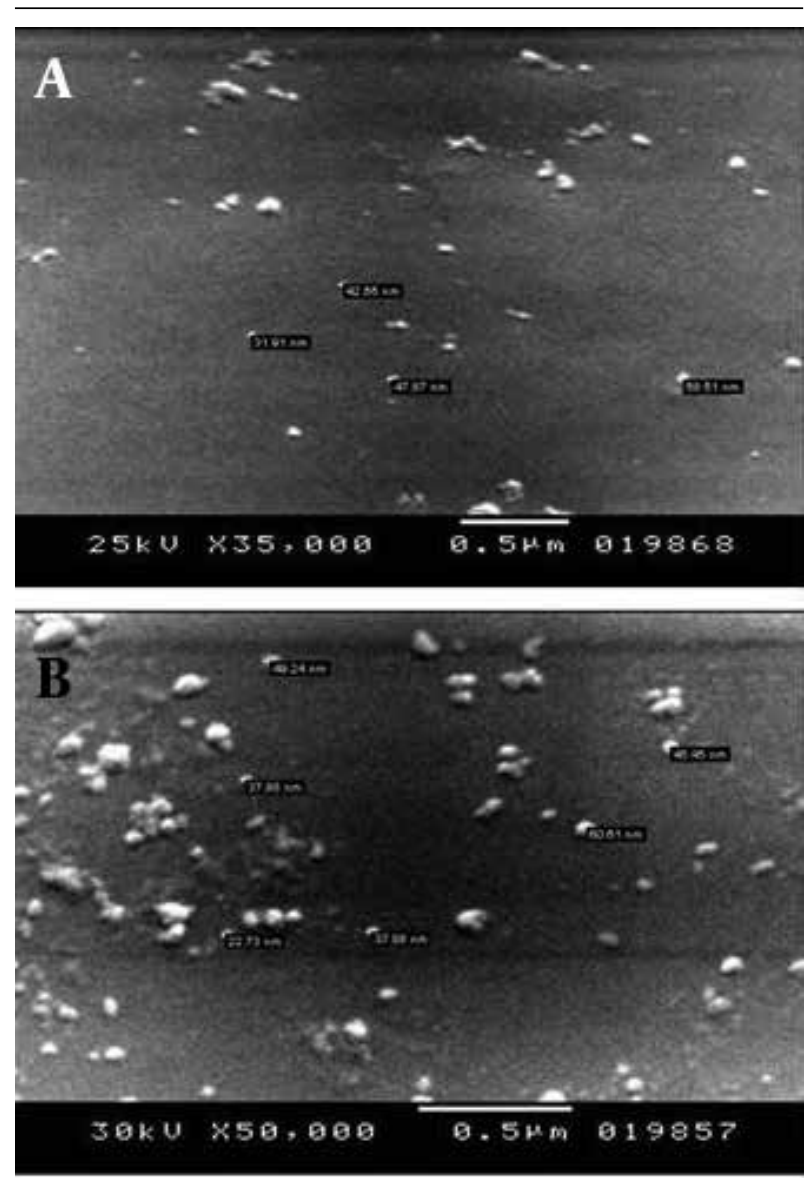

Figure 4. TEM images and diameter vales of synthesized AgNPs from leaf extract of (A) Erucasativa and (B) Spinacia oleracea plants.
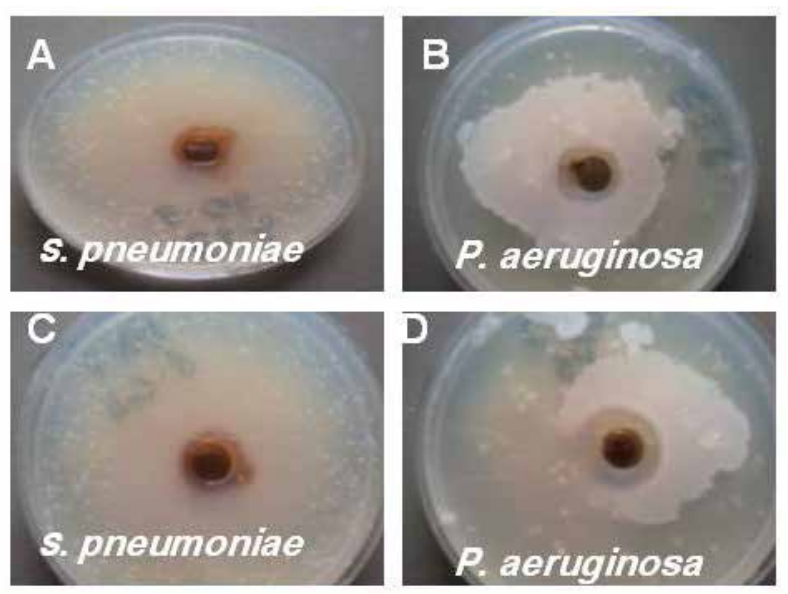

Figure 5. Effect of silver nanoparticles synthesized by leaves extracts of (A and B) Eruca sativa and (C and D) Spinacia oleracea plants on the bacterial growth of S. pneumonia and S. aeruginosa. 


\section{Acknowledgements}

The authors extend their appreciation to the Deanship of the Scientific Research at King Saud University for funding this work through research group no. RGP-VPP 297.

\section{Authors' Contribution}

All authors have participated in the manuscript preparation.

\section{Financial Disclosure}

There is no conflict of interest.

\section{Funding/Support}

The study was self-funded.

\section{References}

1. Joerger R, Klaus T, Granqvist CG. Biologically Produced SilverCarbon Composite Materials for Optically Functional Thin-Film Coatings. Adv Mater. 2000;12(6):407-9.

2. Shankar SS, Ahmad A, Sastry M. Geranium leaf assisted biosynthesis of silver nanoparticles. Biotechnol Prog. 2003;19(6):1627-31.

3. Chandran SP, Chaudhary M, Pasricha R, Ahmad A, Sastry M. Synthesis of gold nanotriangles and silver nanoparticles using Aloe vera plant extract. Biotechnol Prog. 2006;22(2):577-83.

4. Gardea-Torresdey JL, Parsons JG, Gomez E, Peralta-Videa J, Troiani HE, Santiago P, et al. Formation and growth of Au nanoparticles inside live alfalfa plants. Nano Letters. 2002;2(4):397-401.

5. Kagan VE, Bayir H, Shvedova AA. Nanomedicine and nanotoxicology: two sides of the same coin. Nanomedicine. 2005;1(4):313-6.

6. Saxena A, Tripathi RM, Zafar F, Singh P. Green synthesis of silver nanoparticles using aqueous solution of $<\mathrm{i}>$ Ficus benghalensis $<\mid i>$ leaf extract and characterization of their antibacterial activity. Mat Letters. 2012;67(1):91-4.

7. Gardea-Torresdey JL, Rodriguez E, Parsons JG, Peralta-Videa JR, Meitzner G, Cruz-Jimenez G. Use of ICP and XAS to determine the enhancement of gold phytoextraction by Chilopsis linearis using thiocyanate as a complexing agent. Anal Bioanal Chem. 2005;382(2):347-52.

8. Shankar SS, Rai A, Ahmad A, Sastry M. Rapid synthesis of Au, Ag, and bimetallic Au core-Ag shell nanoparticles using Neem (Azadirachta indica) leaf broth.J Colloid Interface Sci. 2004;275(2):496-502.

9. Shankar SS, Rai A, Ahmad A, Sastry M. Controlling the optical properties of lemongrass extract synthesized gold nanotriangles and potential application in infrared-absorbing optical coatings. Chem Mat. 2005;17(3):566-72.

10. Ankamwar B, Chaudhary M, Sastry M. Gold nanotriangles biologically synthesized using tamarind leaf extract and potential application in vapor sensing. Met Organ Nano Met Chem. 2005;35(1):19-26.

11. Shreesh KO, Dharamvir SA. Withania somnifera Dunal (Ashwagandha): A Promising Remedy for Cardiovascular Diseases. World J Med Sci. 2009;4(2):156-8.

12. Mukunthan K, Elumalai E, Patel TN, Murty VR. Catharanthus roseus: a natural source for the synthesis of silver nanoparticles. Asian Pac J Trop Biomed. 2011;1(4):270-4.

13. Malabadi RB, Mulgund GS, Meti NT, Nataraja K, Vijaya Kumar S. Antibacterial activity of silver nanoparticles synthesized by using whole plant extracts of Clitoria ternatea. Res Pharma. 2012;2(4):10-21.

14. Malabadi RB, Meti NT, Mulgund GS, Nataraja K, Kumar SV. Synthesis of silver nanoparticles from in vitro derived plants and callus cultures of Costus speciosus (Koen.); Assessment of antibacterial activity. Res Plan Biol. 2012;2(4):32-42.

15. Xia L, Lenaghan SC, Zhang M, Zhang Z, Li Q. Naturally occurring nanoparticles from English ivy: an alternative to metal-based nanoparticles for UV protection. J Nanobiotechnology. 2010;8:12.

16. Zhang M, Liu M, Prest H, Fischer S. Nanoparticles secreted from ivy rootlets for surface climbing. Nano Lett. 2008;8(5):1277-80.

17. Savithramma N, Rao ML, Rukmini K, Suvarnalatha PD. Antimicrobial activity of silver nanoparticles synthesized by using medicinal plants. International Journal of ChemTech Research. 2011;3(3):1394-402.

18. Savithramma N, Lingarao M, Basha SKM. Antifungal efficacy of silver nanoparticles synthesized from the medicinal plants. Der Pharma Chemica. 2011;3:346-72.

19. Song JY, Kim BS. Rapid biological synthesis of silver nanoparticles using plant leaf extracts. Bioprocess Biosyst Eng. 2009;32(1):79-84

20. Vankar PS, Shukla D. Biosynthesis of silver nanoparticles using lemon leaves extract and its application for antimicrobial finish on fabric. Appl Nanosci. 2012;2(2):163-8.

21. Ghosh S, Patil S, Ahire M, Kitture R, Kale S, Pardesi K, et al. Synthesis of silver nanoparticles using Dioscorea bulbifera tuber extract and evaluation of its synergistic potential in combination with antimicrobial agents. Int J Nanomedicine. 2012;7:483-96.

22. Perez C, Pauli M, Bazerque P. An antibiotic assay by the agar well diffusion method. Acta Biol. Med. Exp. 1990;15:113-5.

23. Cheesbrough M. : Cambridge university press; 2006.

24. Njagi EC, Huang H, Stafford L, Genuino H, Galindo HM, Collins $\mathrm{JB}$, et al. Biosynthesis of iron and silver nanoparticles at room temperature using aqueous sorghum bran extracts. Langmuir 2011;27(1):264-71.

25. Shrivastava S, Bera T, Roy A, Singh G, Ramachandrarao P, Dash D. Characterization of enhanced antibacterial effects of novel silver nanoparticles. Nanotechnology. 2007;18(22):225103.

26. Kim JS, Kuk E, Yu KN, Kim JH, Park SJ, Lee HJ, et al. Antimicrobial effects of silver nanoparticles. Nanomed: Nanotechnol Biol Med. 2007;3(1):95-101. 